\title{
Discussion
}

\section{Exact solutions for the longitudinal vibration of non-uniform rods [J. Sound Vib. 207 (1997) 721-729]}

\author{
Bulent Yardimoglu* \\ Department of Mechanical Engineering, Izmir Institute of Technology, Urla-Izmir, Turkey
}

The aims of this communication are to correct the frequency equation for a free-free rod with area variation $A(x)=A_{0} \sin ^{2}(a x+b)$ in a published article [1] and present the corrected non-dimensional natural frequencies.

Substituting the expression for the displacement of the rod given by Eq. (33) in Ref. [1] into the free-free boundary conditions gives the frequency equation

$$
\tan (k L)=a k[\cot (b)-\cot (a L+b)] /\left[k^{2}+a^{2} \cot (b) \cot (a L+b)\right]
$$

where $k^{2}=\rho \omega^{2} / E+a^{2}$. This is different from Eq. (37) in [1] which we believe is incorrect.

The non-dimensional natural frequencies $(\beta L=\omega \sqrt{\rho / E} L)$ found by solving the corrected frequency equation are given in Table 1 together with the results of Kumar and Sujith [1] for comparison. This shows that the results in Table 6 of [1] are also incorrect. Although not relevant now, the results in Table 6 do not agree even with Eq. (37) in [1].

In Ref. [1], as pointed out by an anonymous reviewer, there is also a misprint in Eq. (34) where the coefficient associated with $J_{-1 / 2}$ should have been $c_{2}$ not $c_{1}$. We also note that while $J_{1 / 2}$ has been defined by Eq. ( 18 ), $J_{-1 / 2}$ has not been defined, but it appears that the correct formula had been used.

Table 1

Comparison of non-dimensional natural frequencies for various values of $a(L=1, b=1)$.

\begin{tabular}{lrrr}
\hline Mode & $a=1[1]$ & $a=1$ Present & $a=2[1]$ \\
\hline 1 & 3.0004297 & 3.309070 & 1.5808147 \\
2 & 6.216901 & 6.375209 & 5.113309 \\
3 & 9.380888 & 9.487363 & 8.436760 \\
4 & 12.533530 & 12.613648 & 11.721540 \\
5 & 15.681720 & 15.745913 & 14.259860 \\
6 & 18.827700 & 18.881240 & 13.317980 \\
\end{tabular}

\section{Reference}

[1] B.M. Kumar, R.I. Sujith, Exact solutions for the longitudinal vibration of non-uniform rods, Journal of Sound and Vibration 207 (5) (1997) 721-729.

\footnotetext{
* Tel.: +902327506781.

E-mail addresses: bulentyardimoglu@iyte.edu.tr, bulyar@hotmail.com.
} 